\title{
Management approach in patient with type 2 diabetes mellitus with bacterial corneal ulcer associated with diabetic keratopathy : a case report
}

\author{
Aditya Riadi Syafei a, Jongky Hendro Prajitno ${ }^{b}$ \\ ${ }^{a}$ Resident of Department of Internal Medicine, Faculty of Medicine Universitas Airlangga,Dr.Soetomo \\ General Hospital, Surabaya, Indonesia \\ ${ }^{b}$ Division of Endocrine, Metabolic and Diabetes, Department of Internal Medicine, Faculty of Medicine \\ Universitas Airlangga,Dr.Soetomo General Hospital, Surabaya, Indonesia
}

\begin{abstract}
Introduction: Diabetes with long standing hyperglycemia commonly causing peripheral neuropathy including corneal nerves. It may lead to delayed diagnosis or underdiagnosed because of asymptomatic due to decreased corneal sensation. Objective: To report a case of secondary bacterial infection of corneal ulcer due to diabetic keratopathy. Case Presentation: A female 63 years old came to ER with a major complaint of pain in her right eye since 2 weeks prior admission. The patient has medical history of long standing diabetes mellitus. She worked as a farmer on rice paddy field. Physical examination in the ER found in the right eye region, positive light perception visual examination, conjunctival hyperemia, subconjunctival abscess, corneal ulcer, hypopyon were found in the anterior chamber of the eye, positive fluorescence test with iris, pupil and lens which is difficult to evaluate. Laboratory result showed mild anemia $(10,6)$, random blood glucose $188 \mathrm{mg} / \mathrm{dL}$, Diabetes Neuropathy Symptom score 2. Discussion: Therapy glycemic control switch from oral to insulin basal bolus subcutan, diet $1800 \mathrm{kkcal}$ per day, gabapentin, antibiotic subconjunctival injection and eye drops, planned for elective cryo-surgery. Patient showed clinical improvement with decreased signs of infection and ocular pain, glucose undercontrolled with insulin. Conclusion: Diabetes mellitus with long standing hyperglycemia should be screened for symptoms of neuropathy. Early diagnosis and treatment could help prevent progressive complications.
\end{abstract}

Keywords: diabetes mellitus, diabetic neuropathy, bacterial corneal ulcer, diabetic keratopathy, case report

\section{Introduction}

Diabetes mellitus (DM) population in the world has increased over the last four decades from 108 million in 1980 to 422 million in 2014 (Bikbova G et al., 2018). According to Basic Health Research (RISKESDAS) data in 2007 with the assumption that Indonesia's population is 230 million, the number of people with DM in Indonesia is 10 million with a prevalence of DM 5.7\% (men 4.9\%, women 6.4\%). In 2013 in Indonesia, there were 8.5 million people with DM (5.5\% prevalence) at that time it was in the 7th position in the world order (Tjokroprawiro A \& Murtiwi S, 2015).

Complications of DM such as nephropathy, retinopathy, neuropathy, and cardiovascular disorders, in which hyperglycemia has an important role. Ophthalmopathy have emerged as a leading cause of blindness in developed countries, where retinopathy is well studied, but contrast to the anterior
IJwW.ijrp.org 
segment ocular complications associated with DM, including the cornea, conjunctiva, and lacrimal gland, there have not been many studies. who studied it, although two thirds of patients reported having diabetic keratopathy during DM (Han BS, Yang HK, Hyon JY, 2019). In fact, of the 382 million people diagnosed with DM worldwide, approximately $70 \%$ suffer from diabetic keratopathy, some type of corneal complication (Vieira-Potter VJ, Karamichos D, Lee DJ, 2016).

DM conditions cause a decrease in the mechanical protective function of the epithelium accompanied by impaired epithelial healing, which increases the risk of ocular surface diseases, such as neurotrophic corneal ulcers, dry eye disease (DED), persistent epithelial defects, recurrent corneal erosions and superficial punctate keratitis (HK, Hyon JY, 2019). In diabetics, it is also found that the adhesion function of the corneal epithelium to the basement membrane is abnormal which can lead to a high risk of corneal infection (Vieira-Potter VJ, Karamichos D, Lee DJ, 2016). In this paper, we will report a case of a patient with type 2 diabetes mellitus who experienced complications of bacterial corneal ulcer related to diabetic keratopathy.

\section{Case presentation}

One patient, Mrs. F, 64 years old, from Gresik Regency, was referred to RSUD Dr. Soetomo Surabaya from RSUD Ibnu Sina with complaints of right eye pain, soreness and decreased ability to see since 2 weeks of SMRS. Initially, the patient complained of a dirty feeling like there was a foreign object in the right eye since 2 days before the patient went to the village nurse. On the previous day the patient was working in the fields pounding rice. At the beginning, the patient did not complain of any pain or stinging sensation in the right eye. The patient then complains of a burning sensation and excessive tearing, especially in the morning and seems to excrete excess feces than usual, accompanied by decreased vision function. The patient was then brought by the family for treatment to the eye clinic at the Ibnu Sina Hospital and was advised to be hospitalized. given therapy by injection of ceftriaxone 1 gram every 12 hours intravenously and mefenamic acid tablets $500 \mathrm{mg}$ orally every 8 hours, injection of subconjunctival amikacin $2 \mathrm{mg}$ every 24 hours. Polyclinic patients treated with $0.5 \%$ moxifloxacin hydrochloride eye drops 1 drop in the right eye every 6 hours, natamycin eye drops 1 drop in the right eye every 6 hours, gentamicin eye drops 1 drop in the right eye every 6 hours, mefenamic acid tablets $500 \mathrm{mg}$ orally every 6 hours. 8 hours. A history of complaints of burning, stabbing or stinging sensations was denied. The patient was then referred to the eye clinic at RSUD Dr. Soetomo for further treatment. 5\% 1 drop right eye every 6 hours, natamycin eye drop 1 drop right eye every 6 hours, gentamicin eye drops 1 drop right eye every 6 hours, mefenamic acid tablet $500 \mathrm{mg}$ orally every 8 hours. A history of complaints of burning, stabbing or stinging sensations was denied. The patient was then referred to the eye clinic at RSUD Dr. Soetomo for further treatment. 5\% 1 drop right eye every 6 hours, natamycin eye drop 1 drop right eye every 6 hours, gentamicin eye drops 1 drop right eye every 6 hours, mefenamic acid tablet $500 \mathrm{mg}$ orally every 8 hours. A history of complaints of burning, stabbing or stinging sensations was denied. The patient was then referred to the eye clinic at RSUD Dr. Soetomo for further treatment.

Past medical history, obtained a history of diabetes which is known since about 20 years ago with a history of treatment that is remembered by the patient's family only glibenclamide and 
metformin tablets obtained from the puskesmas and according to the patient's child there is a lack of medication adherence by the patient. There was no history of high blood pressure, drug allergies. The patient's diet is sometimes 2-3 times a day with vegetables, sometimes mixed with fish, tofu, tempeh and chicken.

Physical examination found good general condition, compost mentis with blood pressure 120/80 $\mathrm{mmHg}$, pulse 90x/minute, lifting strength, regular, respiration $17 \mathrm{x} /$ minute, temperature $36.9 \mathrm{C}$, height $150 \mathrm{~cm}$ and weight approximately $60 \mathrm{~kg}$ with index body mass $22.2 \mathrm{~kg} / \mathrm{m} 2$. Examination of the head and neck revealed no anemia, jaundice, cyanosis, or enlargement of the head-neck lymph nodes and in the right eye region, positive light perception visual examination, conjunctival hyperemia, subconjunctival abscess, corneal ulcer, hypopyon were found in the anterior chamber of the eye, positive fluorescence test with iris, pupil and lens which is difficult to evaluate. Examination of the thoracic region revealed no intercostal retractions, symmetrical chest movements, vesicular breath sounds in both lung fields, no crackles or wheezing were heard. Cardiac examination with the left border of the heart shifted to the left ICS VI midaxillary line, with a single S1-S2 heart sound, no murmurs and gallops were found. Abdominal examination revealed flat, soepel, bowel sounds within normal limits, no enlargement of the liver or spleen was found. Examination of the acral extremities was warm, dry, red, no edema or open lesions were found.

Investigations showed $\mathrm{Hb} 10.6 \mathrm{~g} / \mathrm{dL}$, het $31.8 \%$, leukocytes 10.130/ $\square \mathrm{L}, \mathrm{MCV} 80.3 \mathrm{fl}, 33.3$ g/dL, granulocytes $69.7 \%$, Platelets $273,000 / \square$ L. Glucose 188, BUN 33, SK 0.95, SGOT 15, SGPT 18, Sodium 138, K 4.4, Cl 96, Alb 3.6; PPT 10.7/10.9, APTT 31.2/23.5; HBsAg is non-reactive. From the chest x-ray, the heart appears enlarged with a CTR of $61 \%$ and the lungs do not show infiltrates in both lung fields, both costophrenic angles are sharp. From inspectionThe ECG showed a sinus rhythm tachycardia of 76 beats per minute on the normal frontal-horizontal axis.

The results of the cardiology consultation, the patient was diagnosed with cardiomegaly. Proevaluation was advised to give furosemide $40 \mathrm{mg}$ every 24 hours orally, spironolactone $25 \mathrm{mg}$ every 24 hours orally and elective transthoracic echocardiography. The diagnosis from colleagues was right eye corneal ulcer with hypopyon, cryotherapy was planned under general anesthesia, treated with gentamicin eye ointment every 6 hours right eye, moxifloxacin $0.5 \%$ right eye drop 1 drop every 6 hours, injection of amikacin $20 \mathrm{mg}$ subconjunctiva right eye, atropine eye drops 1 drop right eye every 12 hours.
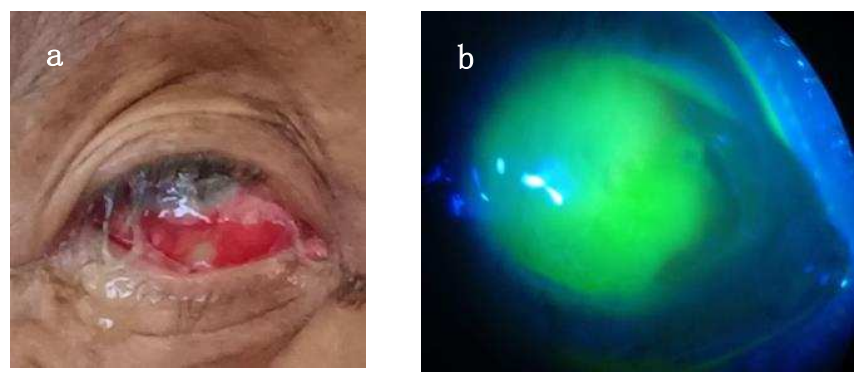

Fig 1. a) Right eye region, positive light perception visual examination, conjunctival hyperemia, subconjunctival abscess, corneal ulcer, hypopyon were found in the anterior chamber of the eye. b) Positive fluorescence test 
From the above data the patient was diagnosed with corneal ulcer with hypopyon, type 2 diabetes mellitus and normochromic normocytic anemia. The patient was programmed diet B 1800 $\mathrm{kcal} / 24$ hours, infusion of $0.9 \% \mathrm{NaCl} 500 \mathrm{ml} / 24$ hours, omeprazole injection $40 \mathrm{mg} / 24$ hours intravenously, mefenamic acid $500 \mathrm{mg}$ every 8 hours orally. Fasting blood glucose diagnostic plan, lipid profile, culture and antibiotic sensitivity test of corneal ulcer swab, elective echocardiography and preparation for surgery.

On the 2nd day, The patient still complains of eye pain and discomfort. No complaints of fever or nausea, vomiting, good appetite. Awareness of compost mentis, blood pressure 120/70 mmHg, pulse $86 \mathrm{x} /$ minute, respiration $16 \mathrm{x} /$ minute, temperature $36.8 \mathrm{C}$. Results of laboratory tests with fasting blood sugar results of $204 \mathrm{mg} / \mathrm{dL}$; blood sugar 2 hours post prandial $288 \mathrm{mg} / \mathrm{dL}$; uric acid $3.7 \mathrm{mg} / \mathrm{dL}$; Na 146, K 3.9, Cl 103, Calcium 8.0; total cholesterol 207 mg/dL; HDL 37 mg/dL; LDL 154 mg/dL; triglycerides $96 \mathrm{mg} / \mathrm{dL}$; serum iron $43 \mathrm{~g} / \mathrm{dL}$; TIBC $204 \mathrm{~g} / \mathrm{dL}$. Our therapy added injection of insulin aspart Novorapid subcutaneously 4 units 15 minutes each before meals and insulin glargine Lantus subcutaneously 10 units at night before bedtime, simvastatin $20 \mathrm{mg}$ every 24 hours at night orally.

On the 4th day, The patient still complains of discomfort in the right eye. Awareness of compost mentis, blood pressure 120/70 $\mathrm{mmHg}$, pulse 86x/minute, respiration 16x/minute, temperature $36.8 \mathrm{C}$. The results of laboratory tests with the results of a basal blood sugar of $307 \mathrm{mg} / \mathrm{dL}$. Our therapy added injection of insulin aspart Novorapid subcutaneously 6 units 15 minutes each before meals and insulin glargine Lantus subcutaneously 12 units at night before going to bed, with a target blood sugar level of 140-180 mg/dL. The patient was planned for cryotherapy surgery under general anesthesia.

On the 7th day, The patient complained of reduced eye pain, swelling of the right eyelid. Awareness of compost mentis, blood pressure 120/70 $\mathrm{mmHg}$, pulse 86x/minute, respiration $16 \mathrm{x} /$ minute, temperature 36.8C. Examination of the status of the right eye region revealed a positive light perception visual examination, palpebral edema, hyperemia in the conjunctiva with minimal secretions with the iris, pupil and lens still difficult to evaluate.

The results of laboratory tests with the results of a basal blood sugar of $108 \mathrm{mg} / \mathrm{dL}$. The results of echocardiography examination revealed left atrial dilatation with an ejection fraction of $71 \%$. The results of swab culture of corneal ulcer lesions found the growth of a culture of Pseudomonas aeruginosa with gram staining found PMN 2+ formation, on $\mathrm{KOH}$ examination no fungal formation was found. The results of the antibiotic sensitivity test were sensitive to amikacin, gentamicin, aztreonam, piperacillin, tazobactam, ceftazidime, ciprofloxacin, levofloxacin, imipenem and meropenem. The diagnosis was right oculi bacterial corneal ulcer with hypopyon. Ophthalmic therapy was replaced with fortified ceftazidime 1 drop in the right eye every 6 hours. Subcutaneous insulin glargine Lantus 8 units at night before bedtime. Cardiological therapy was added with captopril 6.25 mg every 8 hours and bisoprolol 2 ,

On the 10th day,complaints in the eyes are slightly reduced. Awareness of compost mentis, blood pressure 110/60 $\mathrm{mmHg}$, pulse $83 \mathrm{x} /$ minute, respiration $17 \mathrm{x} /$ minute, temperature $36.6 \mathrm{C}$. Examination of the status of the right eye region revealed a positive light perception visual examination, palpebral edema, hyperemia of the conjunctiva with minimal secretions. The results of laboratory tests with the results of basal blood sugar $106 \mathrm{mg} / \mathrm{dL}, \mathrm{Hb} 11.2 \mathrm{~g} / \mathrm{dL}$; leukocytes 8.000/uL; granulocytes $70.8 \%$; platelets $256.000 / \mathrm{uL}$. Polyclinic patients treated with insulin aspart injection 
Novorapid subcutaneous 4 units 15 minutes each before meals and insulin glargine Lantus subcutaneous 10 units at night before bedtime, simvastatin $20 \mathrm{mg}$ every 24 hours at night orally, captopril $6.25 \mathrm{mg}$ every 8 hours and bisoprolol $2,5 \mathrm{mg}$ every 24 hours orally, furosemide $40 \mathrm{mg}$ every 24 hours in the morning, spironolactone $25 \mathrm{mg}$ every 24 hours.

\section{Discussion}

Classification of diabetic neuropathy is generally divided into two, namely symmetrical and asymmetrical neuropathy (Bansal V, Kalita J, Misra UK, 2006). Corneal ulcers can be divided based on three things, namely based on the etiology, ulcer morphology and damage to the corneal layer. Classification based on etiology, divided into two subtypes, namely infectious and non-infectious (sterile). Infections can be caused by bacteria, viruses, fungi and parasites. Non-infection is generally due to systemic connective tissue disease. Classification based on ulcer morphology, namely central or peripheral lesions, presence or absence of purulent secretions, presence or absence of hypopyon (Versura P et al., 2018).

Chronic hyperglycemia is the causative mechanism underlying the pathogenesis of diabetic neuropathy. It induces pathological pathways, such as reactive oxidative stress (ROS) generation, protein kinase $\mathrm{C}$ activation, advanced sorbitol-aldose reductase pathway and advanced glycation end products (AGE) (Nowotny K et al., 2015).

Diabetic corneal neuropathy, corneal fluorescence (accumulation of Advanced Glycation End Products (AGEs)), and a fragile epithelial layer occur cumulatively in diabetic retinopathy (Ljubimov AV, 2017). Several studies have shown that the neuronal component of the retina is associated with the pathogenesis of diabetic retinopathy. Neuronal abnormalities directly affect visual function in diabetic retinopathy, and that neuronal changes may also be the reason for diabetic keratopathy, since the cornea is one of the most heavily innervated tissues (Bikbova $\mathrm{G}$ et al., 2016).

AGE products produced by glycation also play an important role in the pathogenesis of peripheral neuropathy (Aubert CE et al., 2014). Glycation is the nonenzymatic bonding of sugar molecules including glucose or fructose with proteins or lipoproteins, leading to the formation of AGE products with altered structure and function. DM induces myelin protein glycation and deposition of AGE products in perineurial collagen and in the axoplasm of Schwann cells and neurons, leading to microvascular dysfunction, peripheral nerve damage, and axonal degeneration. BS, Yang HK, Hyon JY, 2019).

The increase in intracellular glucose levels caused by chronic hyperglycemia causes a decrease in $\mathrm{Na}+/ \mathrm{K}+$ ATPase activity at the cell membrane, which reduces the speed of nerve conduction and inhibits nerve regeneration (Obrosova IG, 2009). Saturation of the normal glycolytic pathway caused by hyperglycemia in nerve cells results in shunting of excess glucose to the sorbitolaldose reductase pathway, where glucose is converted to fructose and sorbitol by the enzymes sorbitol dehydrogenase and aldose reductase. In the shunted pathway, aldose reductase causes NADPH depletion. The accumulation of fructose and sorbitol leads to a decrease in free neural myoinositol, which leads to reduced membrane $\mathrm{Na}+/ \mathrm{K}+$ ATPase activity. Elevated intracellular glucose levels are also associated with activation of the protein kinase $\mathrm{C}$ pathway, 


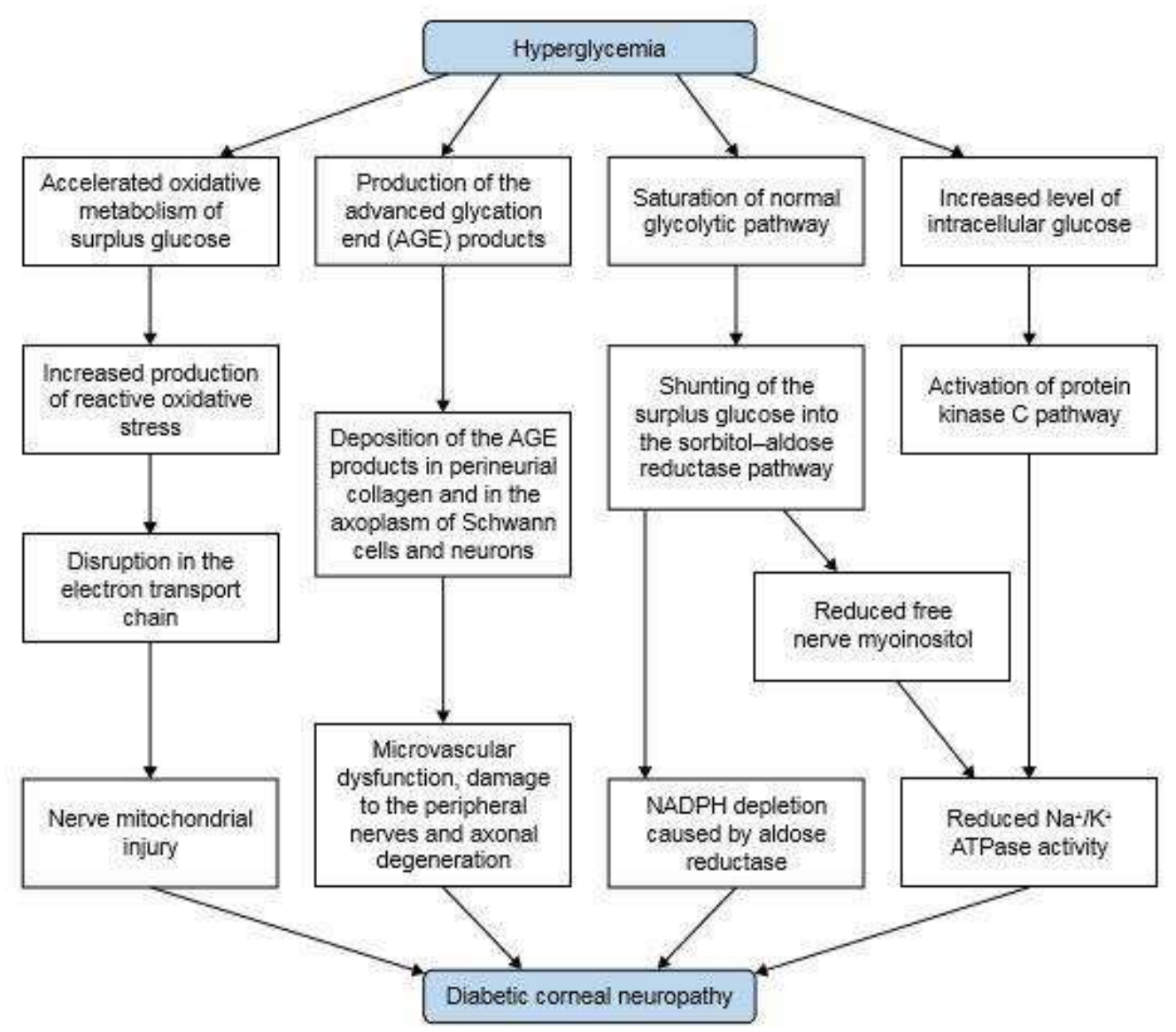

Fig 2. Pathophysiology of diabetic corneal neuropathy (Han BS, Yang HK, Hyon JY, 2018)

Neuropathy is one of common complications of diabetes mellitus, resulted from microvascular injury to the myelinated nerve fibers. The degree of nerve injury in some studies has been associated with the duration of hyperglycemia. Corneal denervation leads to decreased metabolic function, and epithelial cell mitosis, with changes in the epithelial lining including abnormal development of the basal lamina, loss of microvilli, and intracellular edema. Two major reflexes play a role in the mechanical protection of the cornea: the motor function of the blink reflex, and the autonomic function that stimulates tear secretion (Versura P et al., 2018).

Corneal nerves express many epitheliotrophic neuromediators, such as, acetylcholine, substance $\mathrm{P}$, neuropeptide Y, calcitonin-related peptide, noradrenaline, serotonin and vasointestinal peptide. Corneal epithelial cells release a variety of neurotrophic growth factors, including NGF, ciliary neurotrophic factor, and glial cell-derived neurotrophic factor, which are fundamental in ocular surface homeostasis and wound healing. NGF shows an important role in the integrity and function of the ocular surface, stimulating the proliferation and survival of epithelial and nerve fibers (Versura P et al., 2018). 


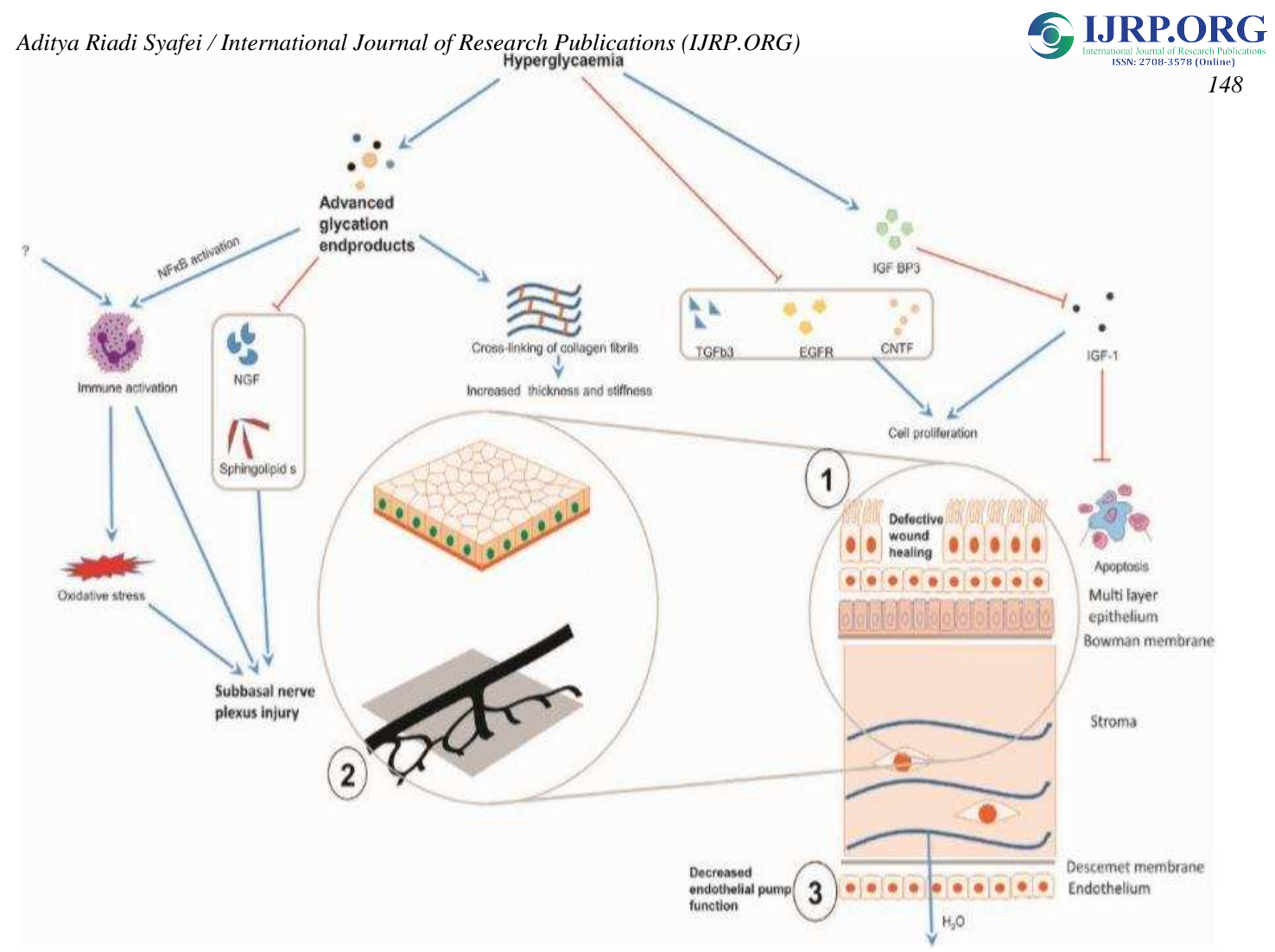

Fig 3. Pathophysiology of diabetic keratopathy (Shih KC, Lam KS-L, Tong L, 2017)

Polymorphonuclear cells (PMN) are secreted in response to disturbances in the cornea, then secrete various lytic enzymes such as collagenase, elastase, and cathepsin that cause damage to the cornea (Vajpayee RB \& Sharma N, 2008). Infection is a secondary process of the occurrence of ulcers on the cornea. Several studies have shown that Pseudomonas aeruginosa and Streptococcus pneumoniae are the main isolates in microbial corneal ulcers in North America (Al-Mujaini A et al., 2009). Prior to initiating antimicrobial therapy, culture is indicated in keratitis of suspected microbial origin. Pathogens can be found by examining the scrapings (scrapping) cornea (American Academy of Ophthalmology, 2018). In this patients with complaints of right eye pain, soreness and decreased ability to see since 2 weeks SMRS feels dirty like there is a foreign object, excessive tears, excessive discharge, accompanied by decreased visual function. History of diabetes since 20 years. Physical examination of the right ocular region revealed positive light perception visual acuity, conjunctival hyperemia, subconjunctival abscess, corneal ulcer, hypopyon in the anterior chamber, slit-lamp test and positive fluorescence test with iris, pupil and lens which were difficult to evaluate.

The first thing in managing all forms of diabetes complications is diet, in addition to tight blood glucose control because hyperglycemia is the main determinant of diabetic microvascular disease (Usuelli V \& Larocca E, 2015). Diet B with a composition of $68 \%$ complex carbohydrates, $12 \%$ protein, $20 \%$ fat, $25 \mathrm{mg} /$ day fiber. Calorie needs are calculated based on body mass index (BMI), which is for body weight or normal BMI, 30 calories per day multiplied by body weight (Tjokroprawiro A \& Murtiwi S, 2015).

Based on the 2015 Perkeni Insulin Consensus guidelines, the use of insulin in hospitalized patients based on the severity of disease, blood sugar targets and monitoring is divided into 2, namely, DM patients with critical and non-critical illnesses. In DM patients with non-critical disease, insulin indications are indicated, among others, not controlled with $\mathrm{OAD}$ and preparation for surgery. Patients who have never received insulin therapy, starting with 5-10 units of prandial insulin and long-acting 
insulin can be given to patients whose blood glucose is controlled day and night but fasting glucose is still high. In this case patients with a history of diabetes treatment with glibenclamide $5 \mathrm{mg}$ and metformin $500 \mathrm{mg}$ every 8 hours but the evaluation of parameters has not reached the therapeutic target. Our therapy added injection of insulin aspart Novorapid subcutaneously 6 units 15 minutes before each meal and insulin glargine Lantus 12 units subcutaneously. Blood sugar at polyclinic was regulated $133 \mathrm{mg} / \mathrm{dL}$. Diet B with 1800 calories per day.

Hypertension is a common comorbidity of diabetes mellitus and a major risk factor for cardiovascular disease and microvascular complications. Blood pressure <140/80 has been recommended for most patients with diabetes mellitus (American Optometric Association, 2014). This patient had no history of high blood pressure or high blood pressure medication. On examination of vital functions, the patient's blood pressure was normotensive 120/80 $\mathrm{mmHg}$.

Patients with diabetes mellitus have a high prevalence of dyslipidemia which affects cardiovascular disease and its complications. LDL target $<100 \mathrm{mg} / \mathrm{dL}$ is recommended in patients without a history of cardiovascular disease, but in patients with a history of cardiovascular disease the recommended LDL level is $<70 \mathrm{mg} / \mathrm{dL}$. Statins (simvastatin, lovastatin, atorvastatin) are used first-line to lower cholesterol(American Optometric Association, 2014). On investigation, this patient's total cholesterol level was $207 \mathrm{mg} / \mathrm{dL}$ and LDL $154 \mathrm{mg} / \mathrm{dL}$. We gave simvastatin $20 \mathrm{mg}$ every 24 hours at night.

Self-monitoring blood glucose systems are starting to be widely used, especially in patients who are dependent on insulin use. Several studies have shown that this system has a positive effect on improving control of patients' blood sugar (American Optometric Association, 2014).

Prophylactic topical antibiotics can prevent ulceration when treatment is started within 24 hours of corneal abrasion (Upadhyay MP et al., 2001). Systemic therapy is useful in cases of scleral, intraocular or systemic infections such as gonorrhea. In certain cases, the choice of initial treatment may be guided by the results obtained from scrapping. Central or severe keratitis (eg, deep stromal involvement or infiltrates greater than $2 \mathrm{~mm}$ with pus), dose every 5-15 minutes followed by frequent application of ointment up to every hour is recommended (American Academy of Ophthalmology, 2018). Single drug therapy using fluoroquinolones has been shown to be as effective as combination therapy using fortified antibiotics by increasing their concentration compared to topical antibiotics (Hanet MS et al., 2012). Topical fortified antibiotics are considered if a visually significant corneal infiltrate is present, especially if hypopyon is present. Ciprofloxacin $0.3 \%$, ofloxacin $0.3 \%$, and levofloxacin 1.5\% have been approved by the Food and Drug Administration (FDA) for the treatment of bacterial keratitis (American Academy of Ophthalmology, 2018). This patient at prior hospital admission received systemic antibiotic therapy of ceftriaxone and moxifloxacin eye drops. While at the Hospital Dr. Soetomo was treated with gentamicin eye ointment every 6 hours in the right eye, moxifloxacin $0.5 \%$ drops in the right eye followed by 1 drop every 6 hours, injection of amikacin 20 $\mathrm{mg}$ subconjunctiva in the right eye.The results of culture scrapping of corneal ulcer lesions found the growth of a culture of Pseudomonas aeruginosa with gram staining found PMN 2+ formation, on $\mathrm{KOH}$ examination no fungal formation was found. The results of the antibiotic sensitivity test were sensitive 
to amikacin, gentamicin, aztreonam, piperacillin, tazobactam, ceftazidime, ciprofloxacin, levofloxacin, imipenem and meropenem.

Topical corticosteroid therapy may have benefit in treating some cases of infectious keratitis, but some literature shows no significant difference in clinical outcomes for interventions with the addition of corticosteroids (American Academy of Ophthalmology, 2018). This patient was not given corticosteroid therapy with consideration at the time with uncontrolled blood sugar, and its administration has not shown clear efficacy.

In patients with diabetic keratopathy, there has been a decrease in the physiological functions of the tropic nerves, one of which stimulates growth hormone which affects the healing time of the corneal epithelium, so periodic and careful evaluation is needed to determine the next step. Evaluation is done every 2 to 4 months on the systemic and local status of the eye (American Academy of Ophthalmology, 2018). Patient at the outpatient clinic in the second month, still had complaints of visual disturbances, and from the results of the examination, ulcer lesions on the cornea without hypopyon were found, and with the results of the randomized controlled patient's blood sugar level examination.

The prognosis of a corneal ulcer depends on the etiology, size, and location of the ulcer and the response to treatment (Byrd LB \& Martin N, 2019). The prognosis for diabetic keratopathy is highly dependent on glycemic control. Managing blood glucose slows progression and improves symptoms of neuropathy (National Institute of Neurological Disorder and Stroke, 2019). Patients with bacterial corneal ulcers that can be associated with diabetic keratopathy, have an ad night dubia prognosis because the level of patient compliance and family support for treatment is not optimal.

\section{Conclusion}

A case about patient with diabetes mellitus who developed a bacterial corneal ulcer in the right eye associated with diabetic keratopathy. Diagnosis of corneal ulcers and diabetic keratopathy in patients is based on history, physical examination and investigations. The patient received subcutaneous insulin therapy to regulate blood sugar, topical and subconjunctival antibiotics, atropine as a cycloplegic and cryotherapy by an ophthalmologist. In the course of the patient's clinical improvement but the ocular status is still difficult to evaluate.

\section{Acknowledgements}

Authors would like to thank to patient, patient's family, staff members of dr Soetomo General Hospital and faculty members of Faculty of Medicine Universitas Airlangga.

\section{Conflict of Interest}

Authors declare that the study was conducted in the absence of any commercial or financial relationships that could be construed as a potential conflict of interest. 


\section{References}

Al-Mujaini A, Al-Kharusi N, Thakral A, Wali UK. 2009. Bacterial keratitis: perspective on epidemiology, clinico-pathogenesis, diagnosis and management. Sultan Qabuus University Medical Journal. 2:184-195

American Academy of Ophthalmology. 2018. Bacterial keratitis preferred practice pattern. Elsevier. 146

American Optometric Association. 2014. Evidence based clinical practice-based guidelines: eye care of the patient with diabetes mellitus. American Optometric Association. 4th eds, p.1-83

Aubert CE, Michel PL, Gillery P. 2014. Association of peripheral neuropathy with circulating advanced glycation end products, soluble receptor for advanced glycation end products and other risk factors in patients with type 2 diabetes. Diabetes Metabolic Research Reviews. 8:679685

Bansal V, Kalita J, Misra UK. 2006. Diabetic neuropathy. Postgraduate Medical Journal. 82(964): 95-100.

Byrd LB \& Martin N. 2019. Corneal ulcer. Florida: StatPearls Publishing, available at : https://www.ncbi.nlm.nih.gov/books/NBK539689/

Bikbova G, Oshitari T, Baba T, Bikbov M, Yamamoto S. 2018. Diabetic corneal neuropathy: clinical perspectives. Clinical Ophthalmology. 12:981-987

Bikkova G, Oshitari T, Baba T, Yamamoto S. 2016. Neuronal changes in the diabetic cornea: perspectives for neuroprotection. Biomed Research International. 1:1-8

Dakhil TAB, Stone DU, Gritz DC. 2017. Adjunctive therapies for bacterial keratitis. Middle East African Journal of Ophthalmology. 1:11-17

Hanet MS, Jamart J, Chaves AP. 2012. Fluoroquinolones or fortified antibiotics for treating bacterial keratitis: systematic review and meta-analysis of comparative studies. Canadian Journal of Ophthalmology. 6:493-499

Han, S. B., Yang, H. K., \& Hyon, J. Y. (2018). Influence of diabetes mellitus on anterior segment of the eye. Clinical interventions in aging, 14, 53-63. https://doi.org/10.2147/CIA.S190713

Ljubimov AV. 2017. Diabetic complications in the cornea. Vision Research. 139:138-152

National Institute of Neurological Disorders and Stroke. 2019. Diabetic neuropathy. Available at https://www.ninds.nih.gov/Disorders/All-Disorders/Diabetic-NeuropathyInformation-Page\#disorders-r3

Nowotny K, Jung T, Höhn A, Weber D, Grune T. 2015. Advanced glycation end products and oxidative stress in type 2 diabetes mellitus. Biomolecules. 5:194-222

Obrosova IG. 2009. Diabetes and the peripheral nerves. Biochim Biophys Acta. 1792:931-940

Oldenburg CE, Lalitha P, Srinivasan M. 2013. Emerging moxifloxacin resistance in Pseudomonas aeruginosa keratitis isolates in South India. Ophthalmic Epidemiology. 3:155-158

Shih, K. C., Lam, K. S., \& Tong, L. (2017). A systematic review on the impact of diabetes mellitus on the ocular surface. Nutrition \& diabetes, 7(3), e251. https://doi.org/10.1038/nutd.2017.4 
Sitompul R. 2018. Corneal neuropathy. In: Proceeding book updates in ocular surface disorders and intraocular infection: risk factors, management and vision optimization. 6:31-33

Stavniichuk R, Shevalye H, Hirooka H, Nadler JL, Obrosova IG. 2012. Interplay of sorbitol pathway of glucose metabolism, 12/15-lipoxygenase, and mitogen-activated protein kinases in the pathogenesis of diabetic peripheral neuropathy. Biochemical Pharmacology. 7:932-940

Tjokroprawiro A, Murtiwi S. 2015. Diabetes mellitus. In: Textbook of Internal Medicine. Surabaya. Airlangga University Press. 2:71-81

Upadhyay MP, Karmacharya PC, Koirala S. 2001. The Bhaktapur eye study: ocular trauma and antibiotic prophylaxis for the prevention of corneal ulceration in Nepal. British Journal of Ophthalmology. 4:388-39

Usuelli and E. La Rocca. 2015. Novel therapeutic approaches for diabetic nephropathy and retinopathy. Pharmacological Research. 98:39-44

Vajpayee RB, Sharma N. 2008. "Pathogenesis of corneal ulceration", in: Taylor HR, Laibson PR, (eds) Corneal ulcers: diagnosis and management. 1st edn. New Delhi: Jaypee Brothers Medical Publishers, pp. 8-12.

Vazirani J, Wurity S, Ali MH. 2015. Multidrug-resistant Pseudomonas aeruginosa keratitis: risk factors, clinical characteristics, and outcomes. Ophthalmology. 10:2110-2114

Versura P, Giannaccare G, Pellegrini M, Sebastiani S, Campos EC. 2018. Neurotrophic keratitis: current challenges and future prospects. Eyes and Brains. 10:37-45

Vieira-Potter VJ, Karamichos D, Lee DJ. 2016. Ocular complications of diabetes and therapeutic approaches. Biomed Research International. 1:1-14 